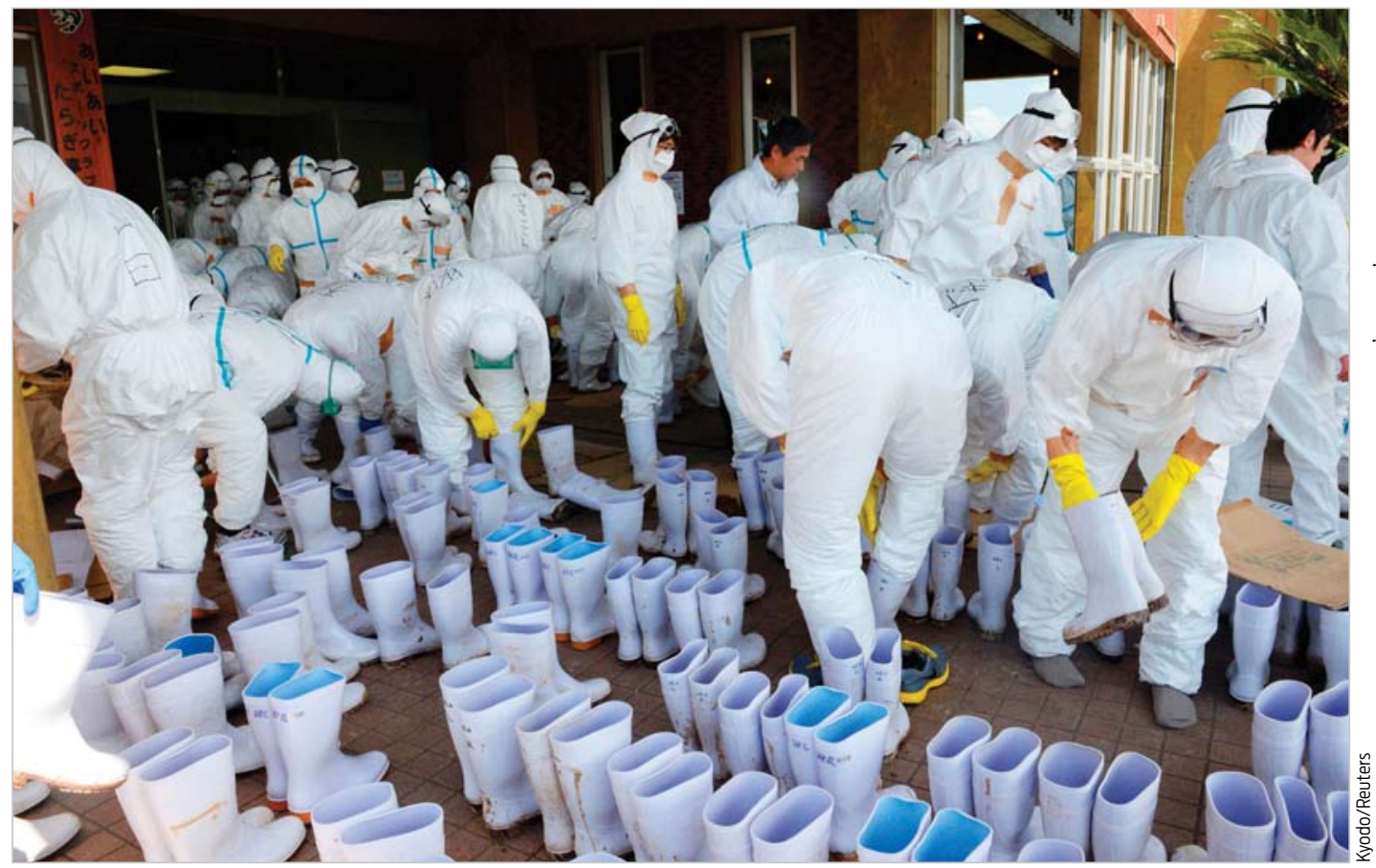

Government employees wearing protective suits put on boots to cull chickens in the Japanese town of Taragi in Kumamoto prefecture on 14 April. Two chickens have tested positive for avian influenza at a farm where more than 1000 chickens have died, marking the country's first case of bird flu in 3 years, the Agricultural Ministry said.

\title{
From NPS MedicineWise
}

\section{Senate Committee recommendations for antipsychotic use in people with dementia}

The recently released Senate Community Affairs References Committee report on the care of Australians living with dementia (http://www.aph.gov.au/ Parliamentary_Business/Committees/ Senate/Community_Affairs/

Dementia/Report/Report) is a positive step towards more judicious use of antipsychotic medicines in people with behavioural or psychological problems associated with dementia.

The report gives 18 key recommendations, including 3-month review by the prescribing doctor to assess the ongoing need for antipsychotic medicines initiated in residents of aged care facilities. Another recommendation would see health professionals undertaking longer consultations with a patient and at least one family member or carer when the patient has presented with dementia. Residential aged care facilities would also be required to report circumstances in which an individual has been prescribed antipsychotic medication for more than 6 months.

While there is sometimes a need for the use of psychotropic medicines, such as antipsychotics, in residents with dementia, Pharmaceutical Benefits Scheme data suggest they are used inappropriately, at doses higher than recommended or in potentially dangerous combinations with other medicines. Utilisation data also suggest extensive off-label use of antipsychotics for patients with dementia in these care settings (http:// www.pbs.gov.au/industry/listing/ elements/dusc-meetings/dos/dusc-dosjun-2013.pdf).

NPS MedicineWise recently collaborated with Webstercare to design a new Quality Use of Medicine (QUM) report within its

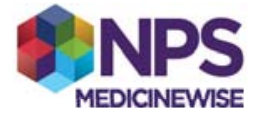

medication management software that helps residential aged care facilities understand and analyse their antipsychotic medicine usage (http://agedcare.nps.org.au). The QUM report flags patients who have been taking antipsychotics for longer than 3 or 6 months, and provides patterns of antipsychotic usage in the residential aged care facility over time. This enables pharmacists to provide information to staff and prescribers working in residential aged care facilities to help them understand, analyse and effectively manage the use of antipsychotic medicines for residents.

Antipsychotics should be used with caution and only when the benefits outweigh the risk of harm. Their use, alongside ongoing nonpharmacological management, also requires careful monitoring, given the serious consequences of getting

doi: 10.5694/mjal4.00561 it wrong.
Lynn M Weekes Chief Executive Officer

NPS MedicineWise 


\section{Impact of antibiotics on growth in undernourished children}

Antibiotics have a growth-promoting effect in young children in low- and middleincome countries, a systematic review and meta-analysis published in the BMJ reports. Ten studies, with data from 4316 children aged 1 month to 12 years), showed an association between antibiotic use and increased mean height or linear growth of $0.04 \mathrm{~cm}$ per month, and an extra $23.5 \mathrm{~g}$ weight gain per month. The authors speculated that the growth-promoting effect of antibiotics might operate through reduction in subclinical infections and beneficial effects on intestinal microbiota. An editorial in the same issue said that the use of antibiotics for promoting growth "poses problems". "Any large scale use of antibiotics must be weighed against the possibility of serious long-term harm both to individuals and to global populations through the emergence of resistance", the editorialist wrote, adding that high-risk children, such as those with defined infections, HIV or severe acute malnutrition, would benefit the most.

BMJ 2014; 348: g2267. doi: 10.1136/bmj.g2267. doi: 10.1136/bmj.g2624
The MJA's trial of the "Comments" section is coming to an end, but there's still time to send us your short comments on any current health care issue, or let us know if you'd prefer to see an increased News section instead.

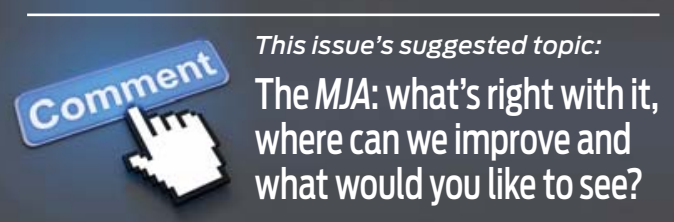

Email us a brief comment (no more than 100 words) on the above question, or any healthrelated subject. Email your comments to: comments@mja.com.au

When sending your comment, please include your full name, discipline and state of residence, and disclose any relevant information or affiliations that may affect interpretation of your comments.

Visit: https://www.mja.com.au/journal/mjainstructions-authors-types-articles-publishedmja\#Comments

\section{Diabetes complication incidence declines as care improves}

Rates of five key diabetes-related complications declined in the US in the past two decades even though the absolute number of cases of complications continues to increase, according to research published in the New England Journal of Medicine. Using data from the National Health Interview Study, National Hospital Discharge Survey, the US Renal Data System and the US National Vital Statistics System, the authors compared the incidences of lower-extremity amputations, end-stage renal disease, acute myocardial infarction (MI), stroke and death from hyperglycaemic crisis between 1990 and 2010. The largest relative decline was seen in acute MI (-67.8\%) and death from hyperglycaemic crisis $(-64.4 \%)$, followed by stroke and amputations $(-52.7 \%$ and $-51.4 \%$, respectively), and the smallest decline in end-stage renal disease $(-28.3 \%)$. The authors said the results probably reflected "a combination of advances in acute clinical care, improvements in the health care system, and health promotions directed at patients with diabetes".

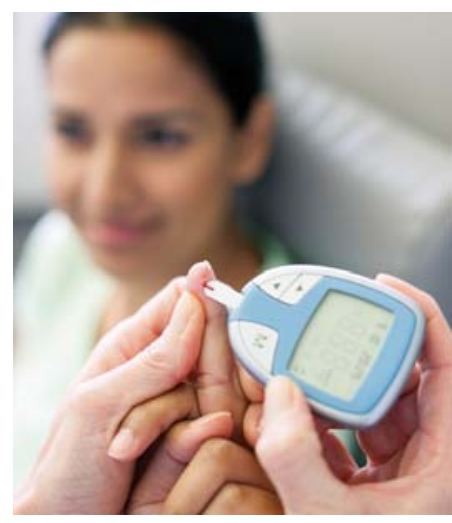

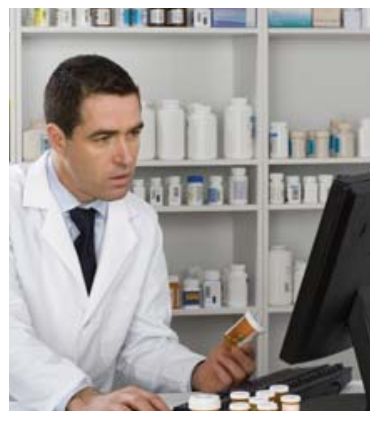

\section{Prescribing pharmacists improve stroke risk factor control}

A nurse-led case management program improved control of key stroke risk factors (hypertension and dyslipidaemia) in patients after minor strokes, but even greater improvements were seen in a prescribing pharmacist-led intervention, according to research published in the CMAJ. None of the 279 participants met guideline targets (systolic blood pressure $\leqslant 140 \mathrm{mmHg}$, fasting low-density

lipoprotein (LDL) cholesterol $\leqslant 2.0 \mathrm{mmol} / \mathrm{L}$ ) at baseline. Participants in both groups received monthly visits for 6 months from either a nurse or a pharmacist. Nurses measured cardiovascular risk factors, counselled patients and faxed results to primary care physicians (active control). Pharmacists did all of the above as well as prescribed according to treatment guidelines (intervention). After 6 months, $43.4 \%$ of participants in the pharmacist group met both systolic blood pressure and LDL guideline targets compared with $30.9 \%$ in the nurse-led group, an absolute difference of $12.5 \%$.

CMAJ 2014; 14 April (online). doi: 10.1503/cmaj.140053
Cate Swannell doi: 10.5694/mjal4.n0505
MJA InSight poll Total respondents:170

Should doctors actively lobby governments about the potential impacts of climate change on public health?

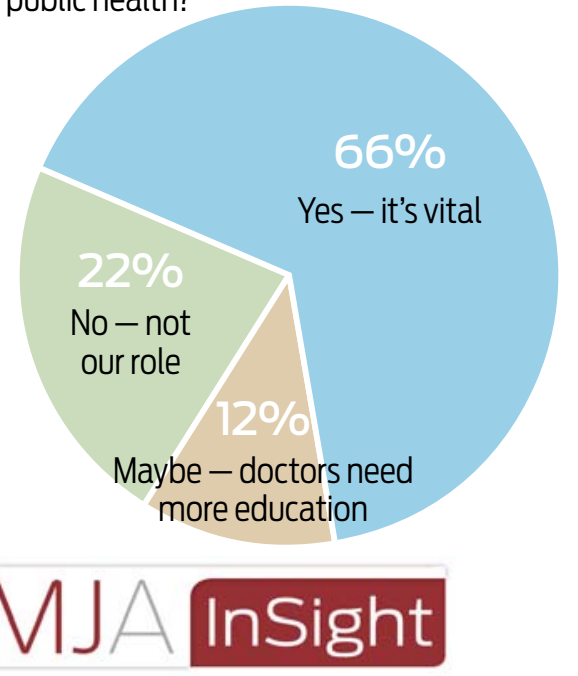

Take part in next week's poll on: www.mja.com.au/insight 